\title{
Heat-Treated Varicella-Zoster Virus Vaccine V212
}

National Cancer Institute

\section{Source}

National Cancer Institute. Heat-Treated Varicella-Zoster Virus Vaccine V212. NCI

Thesaurus. Code C91085.

A heat-treated varicella-zoster virus (VZV) vaccine with potential immunomodulating activity. Upon vaccination with heat-treated varicella-zoster virus vaccine V212, this vaccine may activate the immune system to generate specific anti-VZV antibodies and an active immunity against VZV infection. 